\title{
ERJ
}

Engineering Research Journal

Faculty of Engineering

Menoufiya University

\author{
نحو أنماط سلوكية جديدة لتطوير أنظمة النقل الحضري

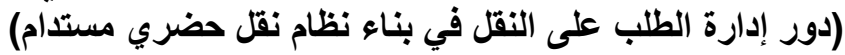 \\ Towards to a New Behavioral Patterns To Development Urban Transportation Systems \\ (The Role of TDM. in the Construction of a Sustainable Urban Transportation System)

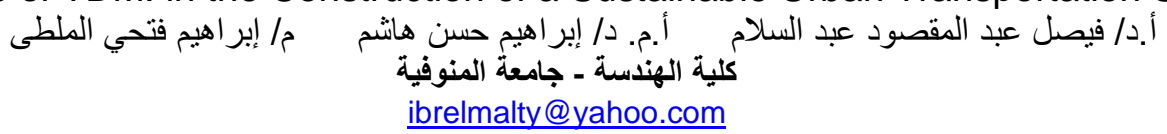

تعتبر عملية تحقيق استدامة أنظمة النقل الحالية من أكبر التحديات التي تواجه معظم المدن الكبرى، لزيادة الكبادة الكفاءة الاقتصادية

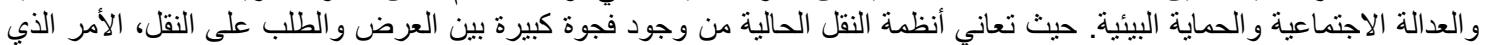

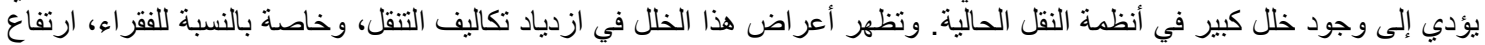

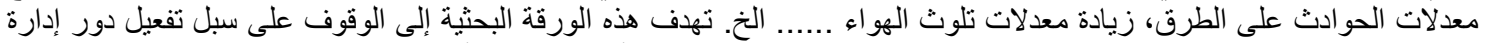

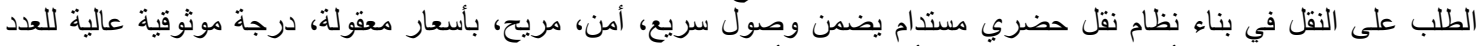

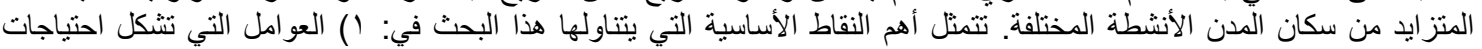

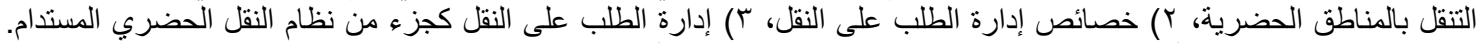

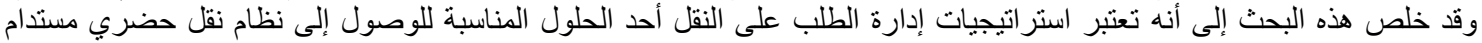
وخاصة بالنسبة للدول النامية بسبب تكاليفها المنخفضة ونئ وفو ائدها المتعددة.

It is the process of achieving the sustainability of the current transport systems of the biggest challenges facing most major cities, to increase economic efficiency and social justice and environmental protection. Current transportation systems between supply and demand suffers from a large gap on the transport, which leads to a significant imbalance in the current transport systems. And show symptoms of this disorder is increasing transportation costs, especially for the poor, high accident rates on the roads, increase air pollution ...... etc. This paper aims to identify ways of activating the role of transport demand management in building a sustainable urban transport system ensures fast access, security, comfortable, affordable, high degree of reliability of the growing number of city dwellers various activities. The most important key points covered in this research in the factors that make up the mobility needs of urban areas, the demand for transport management properties, and transport demand management as part of sustainable urban transport system.

الكلمات المفتاحية: الطلب علي النقل - إدارة الطلب - النقل المستدام.

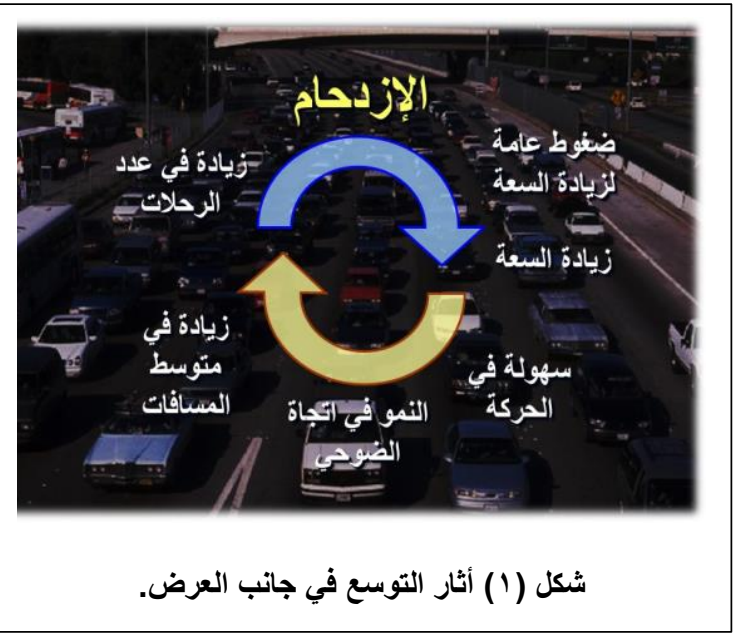

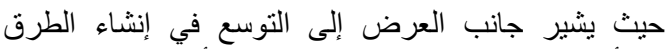

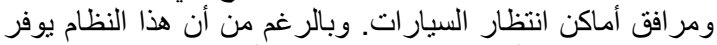

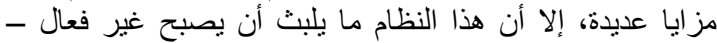

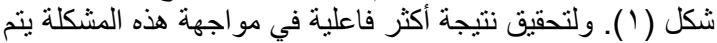

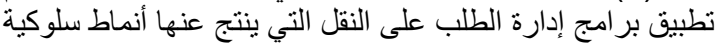

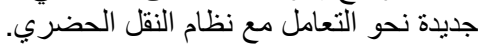

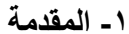
إنَّ النمو السكاني و التوسُع العمر اني الذي شهدته معظم المدن

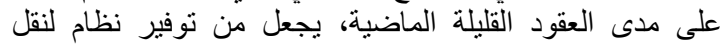

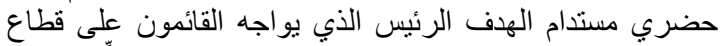

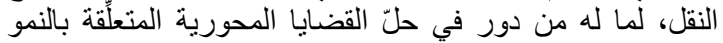
الحضري واستخدامات الأر اضي و استهلاك الطاقة وتغيّر المناخ. فلا يخفي علي أحد الطبيعة الغير مستدامة لأنظمة النقل

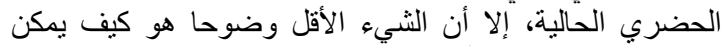
التحرك نحو مستقبل أكثر استدامة في مجاء الحئ النقاء النقل الحضري.

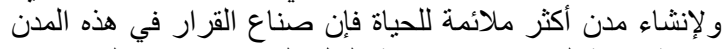

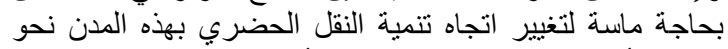

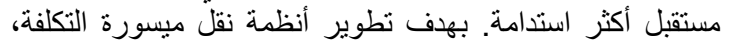
مجدية اقتصاديا، موجهة للناس، وصديقة للبئئة

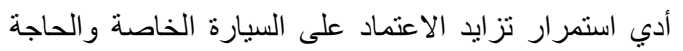

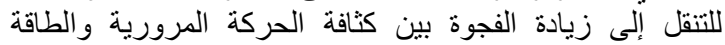

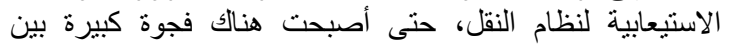

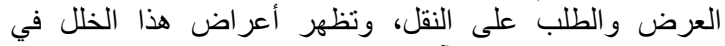

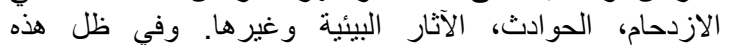

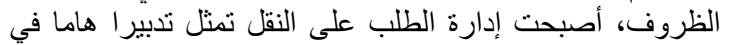

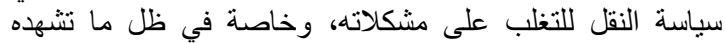

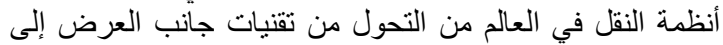
إدارة الطلب. 
أ.د/ فيصل عبد الدقصود عبد السلام أ.م. د/ إبر/هيم حسن هاشم م/ إبراهيم فتحي الدلطى " نحو أنداط سلوكية..."

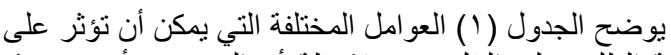

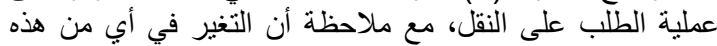

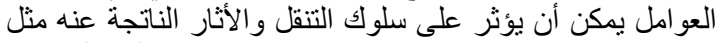

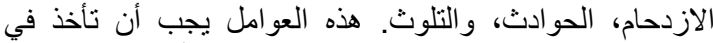

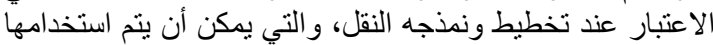
لإدارة عملية الطلب على النقل.
Transportation Demand الطب على النقل

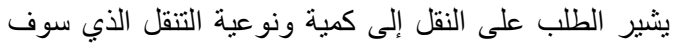

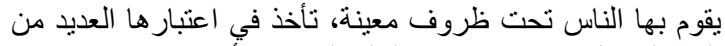

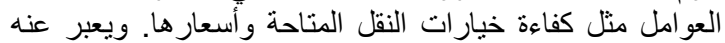

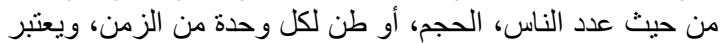
فهم الطلب على النقل من العو امل المهمة لعملية تخطيط النقل.

\begin{tabular}{|c|c|c|c|c|c|}
\hline الايموغر افيا & الاقتصاد & الأسعار & خيارات النقل & كفاعة الخدمة & الأر اضتعمالات \\
\hline 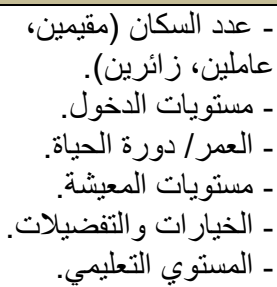 & - ـ ـ الدخد الوظائف. & 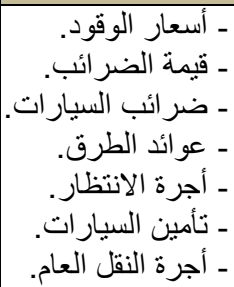 & 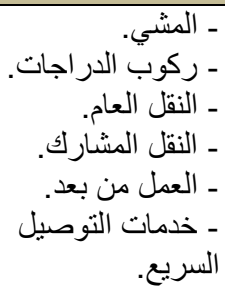 & 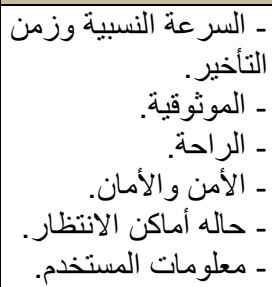 & ـ ـ ـ ـ الاكثافة. \\
\hline
\end{tabular}

جدول (1) العوامل التي تؤثر على الطلب على النقل.

Hester, R.E. and Harrison, R.M., "Transport and the environment", The Royal Society of Chemistry, 2004.

r-r r عوامل النمو في الطلب على النقل.

تحدث زيادة الطلب على النقل لسببين أساسيين هما: زئل

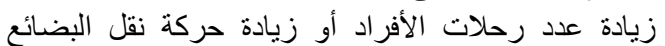

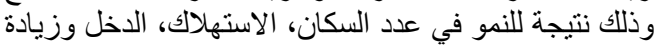
الإنتاجية الاقتصادية.

النمو في منوسط المسافة سواء بالنسبة لحركة الركاب أو الراب حركة البضائع. هذان العاملان غالبانع ما يحدثان بصورة متزامنة الأمر الذي الذي

ينتج عنه زيادة في حجم الطلب على على النقل. ويمكن تقسيم العو امل التي تؤثر في حجم الطلب على لقى النقل إلى التالي ()

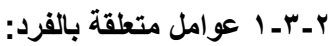

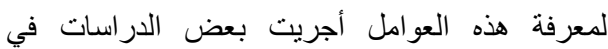

التسعينيات لمعرفة الأسباب الحقيقة لتحرك الترك الناس أي "الدو افع" فتوصلو ا إلى النتائج التالية: يزداد عدد التحركات بزيادة الدخل. يزداد عدد التنقلات حسب وسيلة النقل. تؤُثر الفئة الاجتماعية على التنقلات.

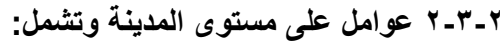

نمط التخطبط في المدينة (مركزي - شبكي .....) يؤثر في هوني

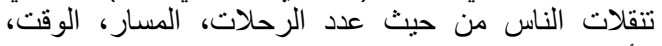

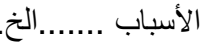
البعد والقرب من مراكز المدن "تزداد عدن التزب التنقلات كلما

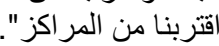

الكثافة السكانية " تقل الحركة كلما زادت الكثافة و العكس".

T- إدارة الطلب على النقل TDM

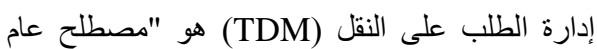

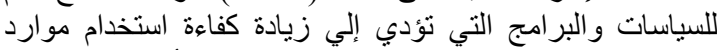

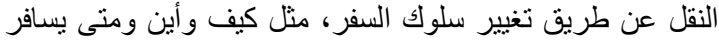

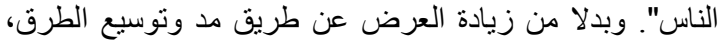

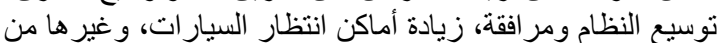
المرافق الأخرى. فإن إدارة الطلب على النى النقل تؤكد على حركة ولى

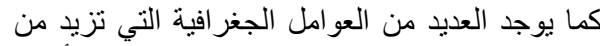
ذجم الطلب على النقل منها الموقع بالضواحي، الإقامة في أحياء

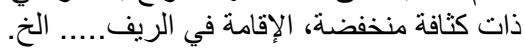
r ـ ا 1 متغيرات الطلب على النقل.

تختلف متغيرات الطلب على النقل وفقا لطبيعة ما يتم نقله

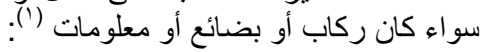

• الركاب Passengers: يمثل الطلب على النقل دالة

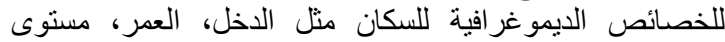

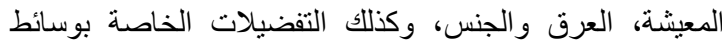
النقل المختلفة (عام - خاص - مشاة - دراجن الجات......).

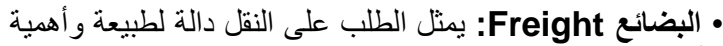
الأنشطة الاقتصادية (الناتج المحلي الإجمالي، المسطحات التجارية

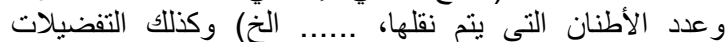
الخاصة بوسائط النقل المختلفة. وعملية تقييم الطلب علي علي نقل

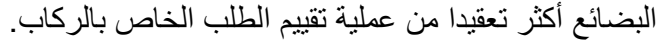

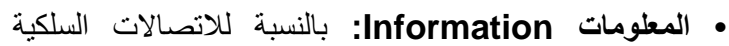

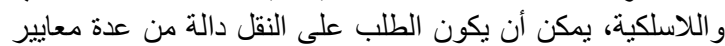

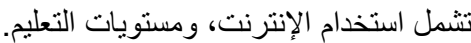

r-r العوامل التي تشكل احتياجات الطلب على النقل بالمناطق الحضرية

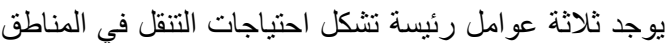

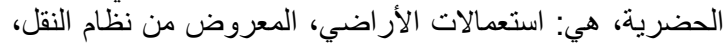

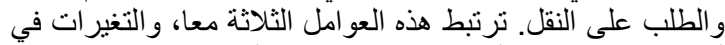

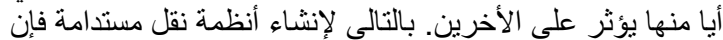

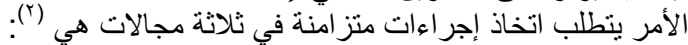

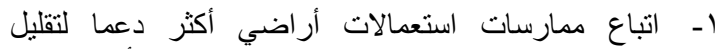

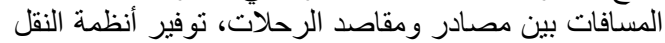

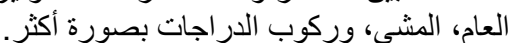

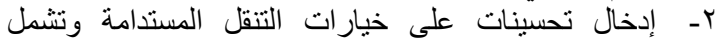

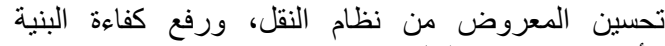
الأساسية وخدمات النقل

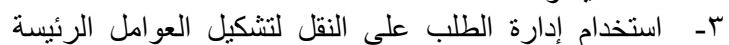

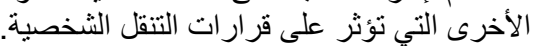


أ .د/ فيصل عبد الدقصود عبد السلام أ.م. د/ إبراهيم حسن هاشم م/ إبر/هيم فتحي الهلطى " نحو أنعاط سلوكية..."

كما حدد المبادئ التصميمية لإدارة الطلب على النقل في أربع مبادئ رئيسة هي:

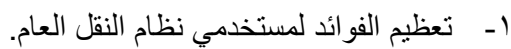

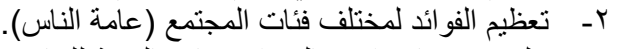

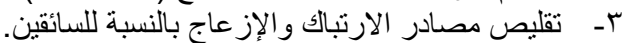

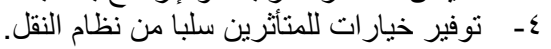

r-1 أسباب إدارة الطلب على النقل

إدارة التنقل (الطلب على النقل) هو استجابة عامة لمشاكل

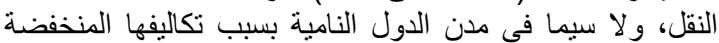

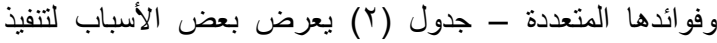
إدارة الطلب على النقل في البلدان النامية.
الناس والبضائع وليس السيارات فقط وتعطي الأولوية في ذللك لوسائل النقل العام (q).

وقد اقترح Rosen-bloom (1998) أربع فئات أساسية

لإدارة الطلب على النقل هي (19) (19):

ـ الحد من عدد المركبات بزيادة الإشغال.

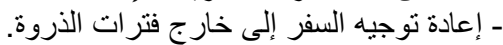

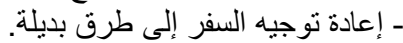
ـ الحد من إجمالي الطلب على السفر.

\begin{tabular}{|c|c|}
\hline الوصف & العامل \\
\hline 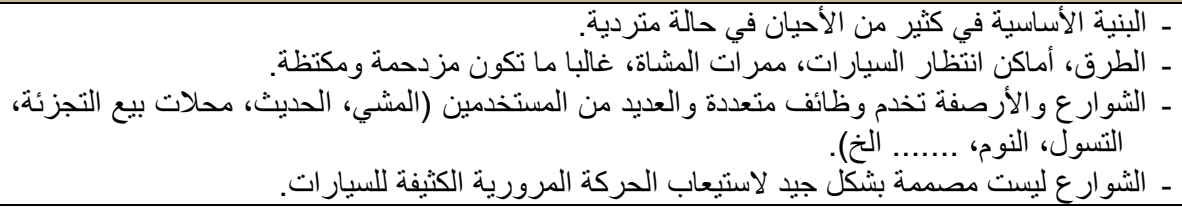 & إلأساسية. البنية \\
\hline 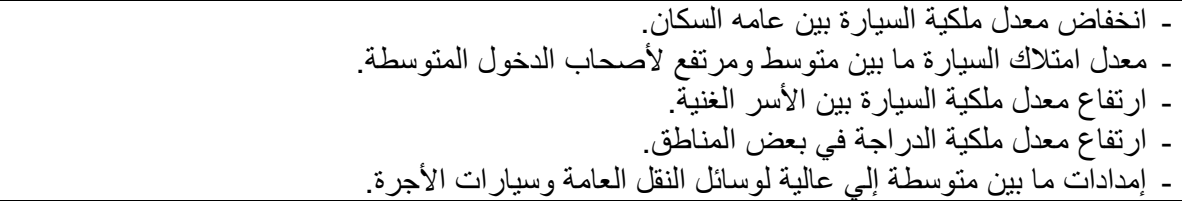 & المعروض من المبات \\
\hline 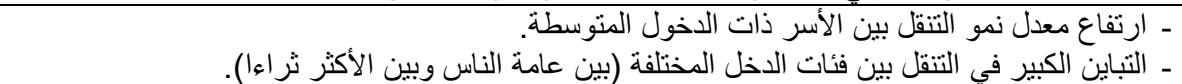 & التنقل الشخصي \\
\hline 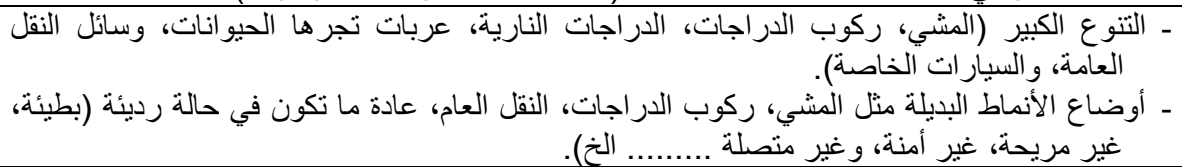 & تنوع أنماط النقل \\
\hline 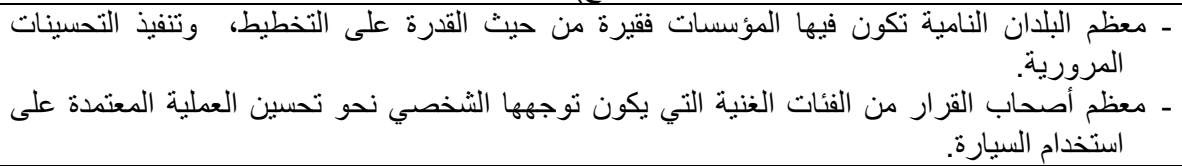 & لمؤسسية \\
\hline ـ ـ تمويل محددو للبنية التحتية للنقل و الخدمات. & التكاليف الحكومية \\
\hline ـ كثير من الأسر تنفق جز عا كبير ا من دخلها على النقل. & تكاليف المستخدم \\
\hline 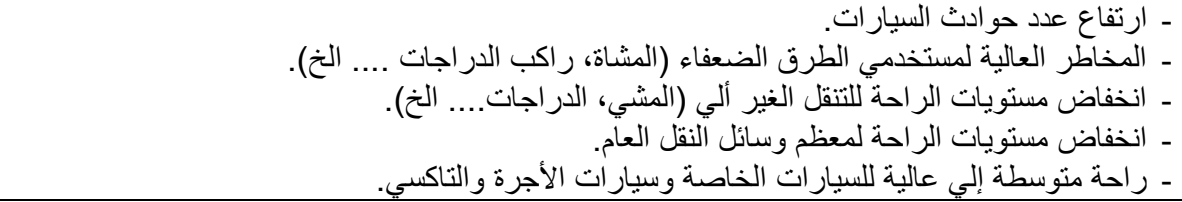 & السلامة المرورية المرية \\
\hline ـ ـ تركز عالي للتلوث بالمناطق الحضرية. & البيئة \\
\hline 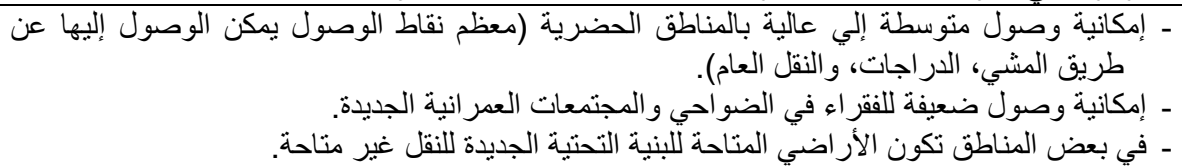 & خي \\
\hline 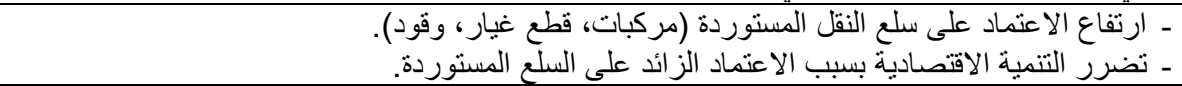 & التنمية الاقتصادية التية \\
\hline
\end{tabular}

جدول (r) العوامل التي تبرر إدارة الطلب على النقل في البلان النامية.

Source: Monowar Mahmood, Traffic Management System and Travel Demand Management (TDM) Strategies:, Asian Journal of Management and Humanity Sciences, Vol. 4, No. 2-3, pp. 161-178, 2009. 
أ .د/ فيصل عبد الدقصود عبد السلام أ.م. د/ إبراهيم حسن هاثم م/ إبر/هيم فتحي الهلطى " نحو أنعاط سلوكية..."

لذلك فإن إدارة الطلب على النقل هي الحل المناسب لمشكلات النقل بالدول النامية.

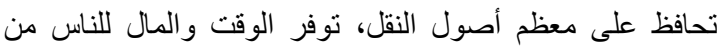

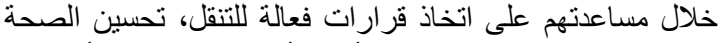

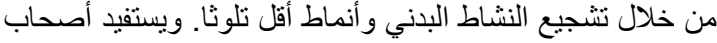

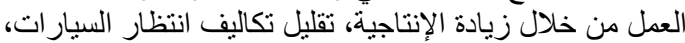

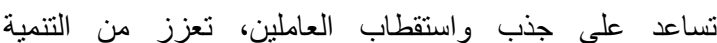
الاقتصادية عن طريق الحد من الازدحام وتحسين الحركة.

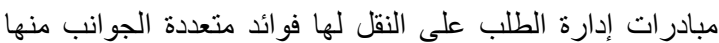
تو افر بدائل للقيادة كجزء مل من الخيار ات المناحة.

تعمل إدارة الطلب على النقل على المستوي الفردي، ولكن لديها فيهاء

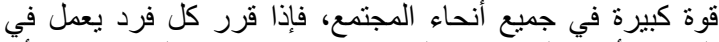

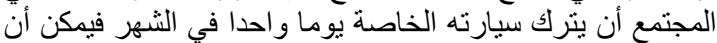

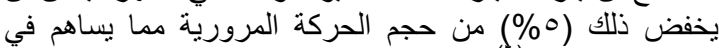

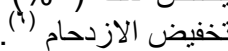

r-r استراتيجيات إدارة الطلب على النقل

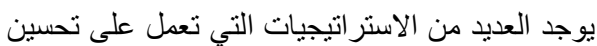

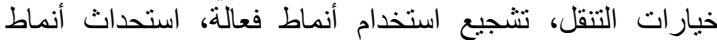

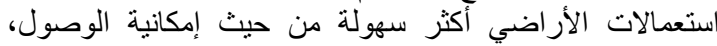
وإصلاح الممارسات التخطيطية المتحيزة لنمط معين (الحركة الترانة

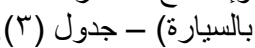

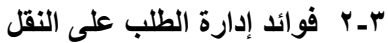

تتمثل أهم نتائج إدارة الطلب على النقل في "أنماط سلوكيه

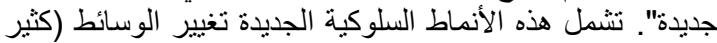

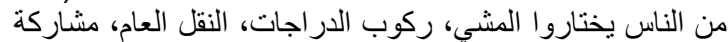

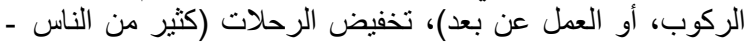

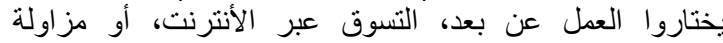

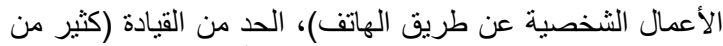

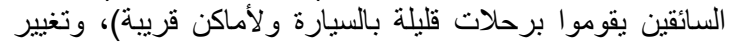

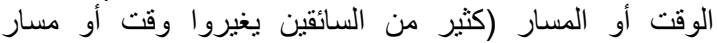
رحلاتهم لتجنب الازدحام المروري).

وبالتالي تتمثل الفوائد الرئيسة لإدارة الطلب على النقل في التالي:

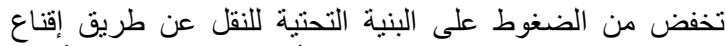

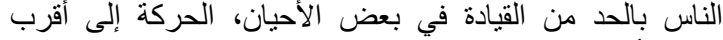

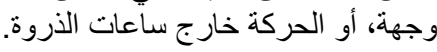

تجعل قرارات السفر الخاصة أكثر فاعلية، كثير من السائقين

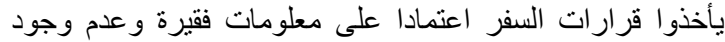

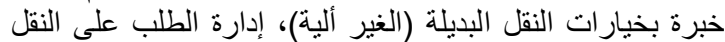
تحسن من و عيهم بالخيار ات البديلة.

تعظم العائد من الإنفاق على البنية التحتية، وقد أظهرت النهات الدراسات

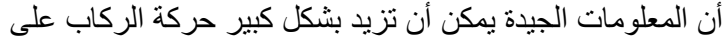

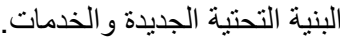

\begin{tabular}{|c|c|c|c|}
\hline البرامج وإصلاح السياسات. & تحسين خيارات التنقل. & $\begin{array}{c}\text { التدابير الاقتصادية من القيادة) } \\
\text { لحوافز }\end{array}$ & إدارة استعمالات الأراضي \\
\hline 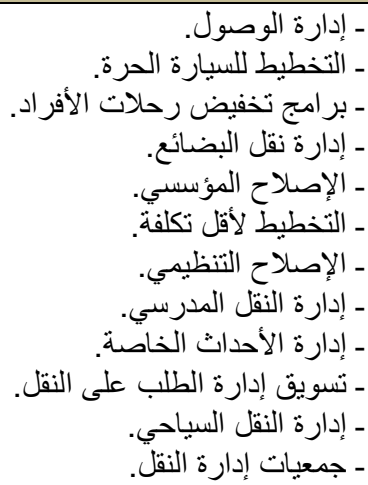 & 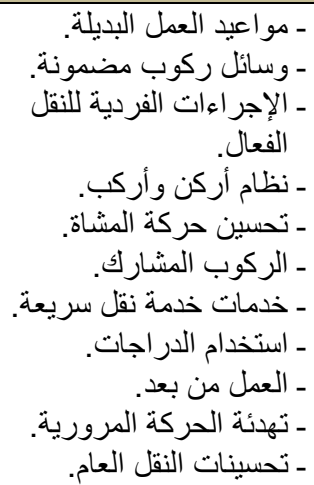 & 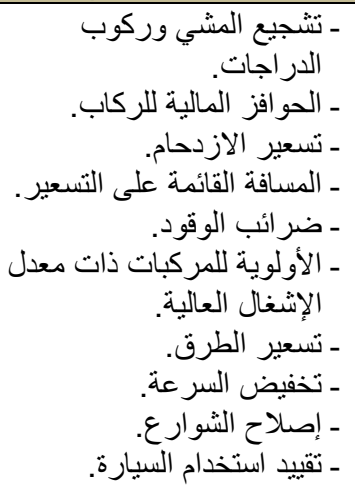 & 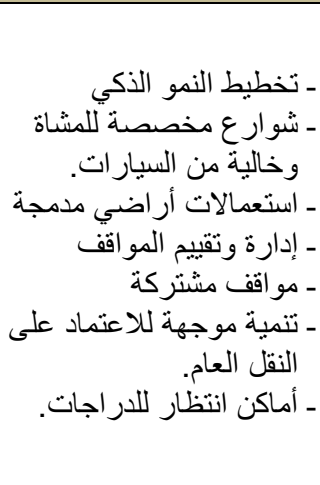 \\
\hline
\end{tabular}

جدول (r) استراتيجيات إدارة الطلب على النقل.

Source: Victoria Transport Policy Institute, Overview of TDM Strategies and Their Stakeholder - 2014.

مر اعاة أن بعض التغيرات في التنقل تؤدي إلى انتعاش في مزي

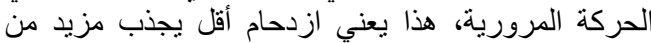
السيار ات الخاصة ويزيد من أطوال الرحات الرحات.

قياس الأثار الناجمة عن التغير في أنماط التنقل عن طريق الإني

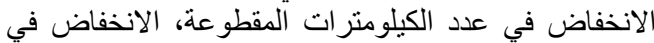
نسبة مشاركة السيار ات الخاصة في التنقل، الزيادة في نسبة الإنة مشاركة النقل الغير ألي والنقل العام في التنقل.

مراعاة الفوائد المختلفة لاستراتيجيات إدارة الطلب.

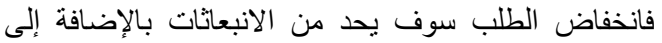

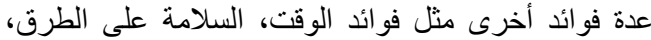

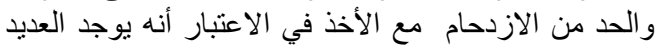

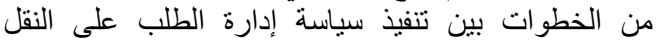

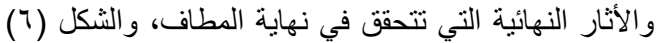

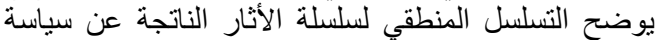
إدارة الطلب على النقل للتقليل من الانبعاثات، حيث يلاحظ النّاته r- ـ خطوات صياغة سياسات إدارة الطلب على النقل

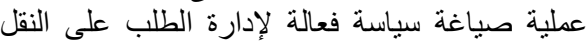

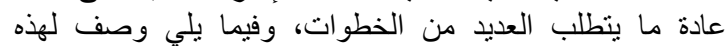
الخطوات و العقبات المشتركة و المخاطر التي يجب ولبي التخلب عليها:

البدء مع أهداف محددة بوضوح (ما تريد تحقيقه في نهاية

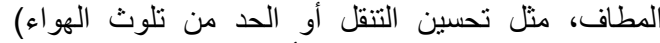

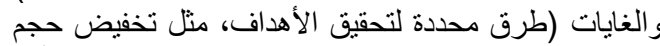
حركة مرور السيارات وإدخال المركبات النظيفة الأقل

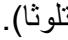

تحديد سياسات إدارة الطلب على النقل، مثل تحسين أنماط

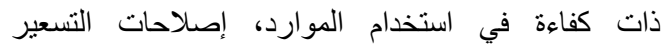

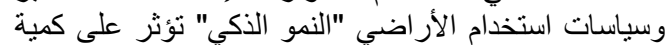
ونوع التنقل الذي يختاره الناس. 
أ .د/ فيصل عبد الدقصود عبد السلام أ.م. د/ إبراهيم حسن هاشم م/ إبر/هيم فتحي الهلطى " نحو أنعاط سلوكية..."

الخاصة لمختلف أغراض التنقل. كما يقلل من نسبة

(الانبعاثات).

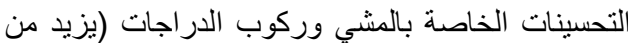

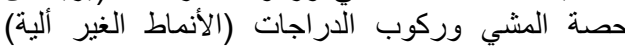

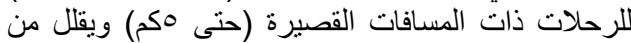

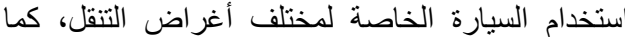
يقلل من نسبة انبعاثات الغاز الخات.

ثانيا: القيود والحوافز اللازمة للحد من استخدام السيارات تسعير الطرق و الازدحام (يقلل من التنقل بالسيارة خلال ساعات الذروة، ويخفض من الازدمام (لانبعاثات).

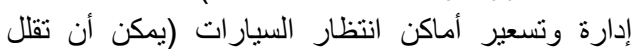

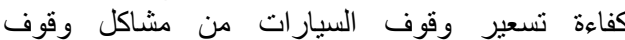
السيار ات، وتثجيع استخدام وسائل النقل البديلة).

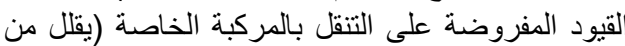

التنقل بالسيارة في أوقات معينة أو في مناطق معينة).

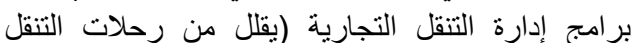

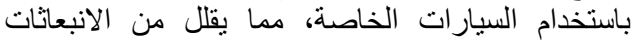

وخاصة في حالة مشاركة أرباب العمل الرئيسين).

ثالثا: تطوير واستخدام الأراضي في المناطق الحضرية

تشمل سياسات استعمالات الأر اضي الكثيفة و المتداخلة

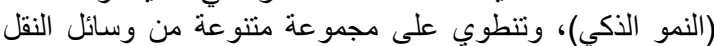

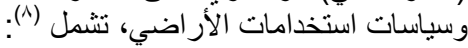

التخطيط لاستراتيجي: وضع رؤية مجتمعية شاملة توجه

قرارات استخدامات الأراضي والتيع رالنقل الفردية. على سلى سبيل

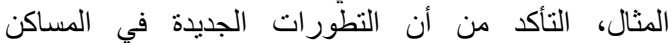

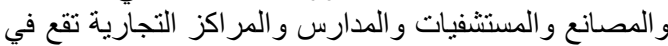
أو بالقرب من المدن القائمة، على مسار الت النقات النقل العام الرئيسة بدلا من المنتشرة في أنحاء الريف.

التمية الكثيفة للمناطق الحضرية بدلا من التتمية المنتشرة: تقارب الأنشطة وتمركز ها.

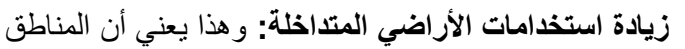

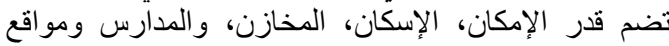
العمل والترفيهية وتقع قريبة من بعضهان الإنهان البعض، داخل ومل الأحياء نفسها.

تثجيع أنواع متنوعة من المساكن وكذلك الأسعار: تطوير

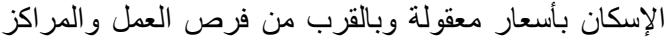

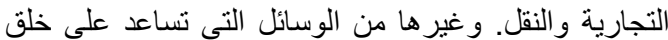
المزيد من المساكن بأسعار معقولة.

خلق مجتمعات مكتفية ذاتيا: خفض منوسط مسافات الرحلة،

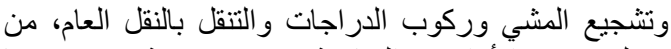

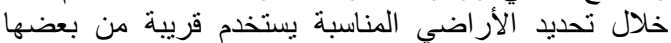

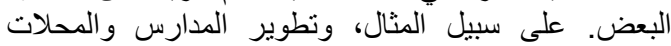

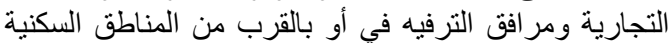

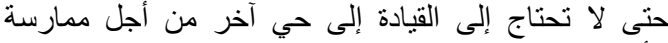
الأنشطة الرياضية المدرسية، و التسوق.

• تحسين ظروف التنقل عبر الأنماط الغير آلية: تحسين

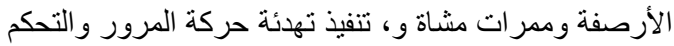

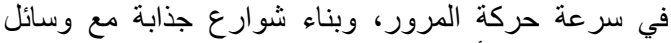
الراحة للمشاة (الأشجار و المظلات، ....... الخ.

• تثجيع التنمية الموجهة للاعتماد على النقل العام: زيادة

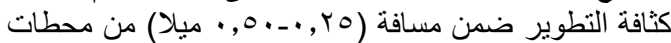

أن تغير السياسة بغير من تفضيلات خيار النقل مما يغير في

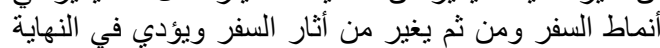

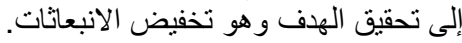
rــ تـابير إدارة الطلب على النقل

تتكون إدارة الطلب على النقل من عدد كبير من التدخلات الصغيرة التي يمكن أن تؤثر بشكل تر اكمي على على استعمال السيارة،

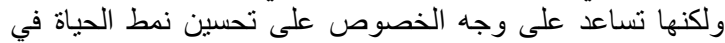
المدن. كما يمكن من خلالها تغيير سلوك التنقل لزيادة كفاءة نظام

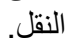

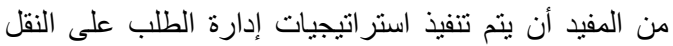

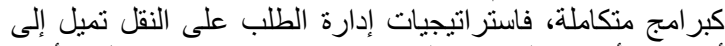

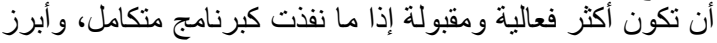

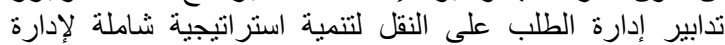

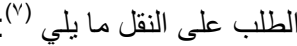

أـ نهج الدفع والجذب Push - and Pull Approach. يؤكد هذا النهج على أن التدابير الخاصة بالنقل الحضري

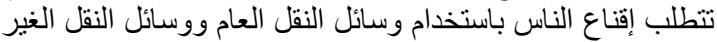

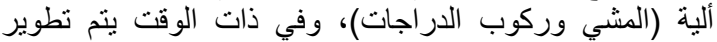

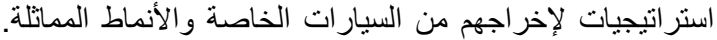

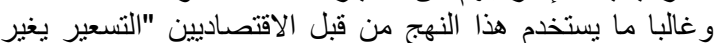

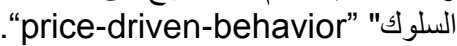

ب- النقل العام، النقل الغير آلي، والتنمية الموجهة للنقل العام. يتم هذا النهج من خلال تتفيذ التدابير الثلاثة التالية:

-

ينطوي هذا الإجر اء على تطوير أنظمة نقل عام ذات جودة

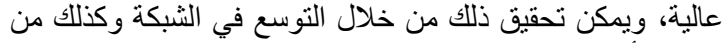
خلال الأنظمة متعددة الوسائط.

-

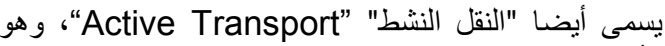
شير أساسا إلى المشي وركوب الدراجات، وكذلك البنية التحتية

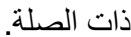

\section{- -} .Development

يشير إلي النهج الخاص بالتصميم الحضري حيث يتم تعزيز

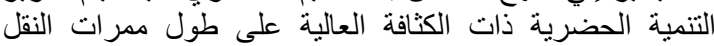
الجماعي (سيرفيرو - 1998 1ل ).

ج- التجنب، التحول، التحسين Avoid, Shift, Improve. النهج الثالث يتم من خلال تنفيذ التدابير الثلاثة التالية:

التجنب: تسعى هذه الاستراتيجية إلى تجنب السفر غير الضروري

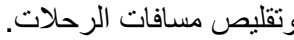

التحول: تسعى هذه الاستر اتيجية إلى تحويل الركاب نحو مزيد من

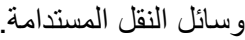

التحسين: تركز على السياسات التي تهدف إلى تحسين ممارسات النقل و استخدام التكنولو جيا.

نجاح هذه التدابير ينطلب التحرك على ثناثة محاور تشمل: أولا: توفير وسائط عالية الجودة من حيث معدل الانبعاثات والبنية التحتية (سحب) تشمل:

• تحسينات خدمة النقل العام (من شأنه أن يزيد من معدل استخدام وسائل النقل العام ويقلل من استخدام السيارة 
أ .د/ فيصل عبد الدقصود عبد السلام أ.م. د/ إبراهيم حسن هاشم م/ إبر/هيم فتحي الهلطى " نحو أنعاط سلوكية..."

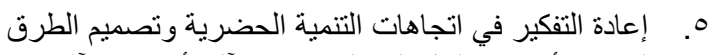
لتحسين أوضاع النقل المختلفة سواء الآلية أو غير الآلية الهية.

V-r إدارة الطلب على النقل كجزء من نظام النقل المستدام

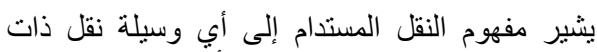

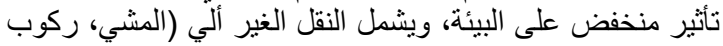

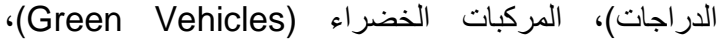

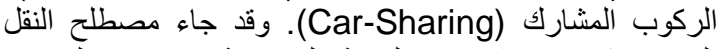

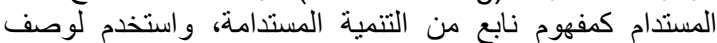
وسائل النقل، ونظم تخطيط النقل التي تنفق مع الهتمامات

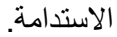

وقد أشار مركز النقل المستدام الأوربي عام 991 ام النقام إلى أن نظام النقل المستدام هو النظام الذي يتصف بالتنالي:

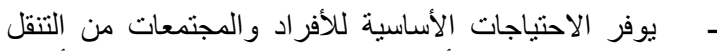

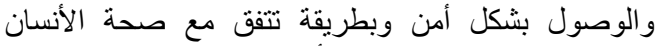
و البيئة، و العدالة ضمن ولئل المن وبطر الأجيال.

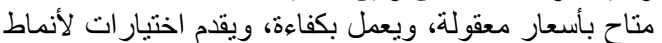

النقل العام والممرات ذات القدرة العالية، وتوفير مرافق المشاة و الدر اجات ذات جودة عالية في تللك المناطق.

تطوير شبكات طرق ومسارات متصلة: إنشاء شوارع

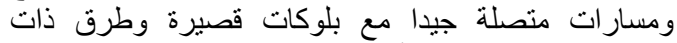
نهايات مغلقة عند الحد الأدنى.

• المحافظة على الثوارع الضيقة: استخدام إدارة المرور

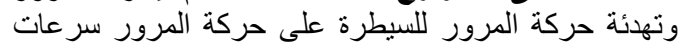

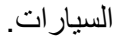

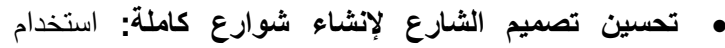

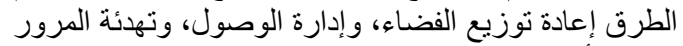

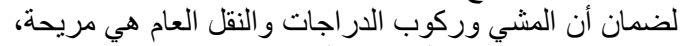

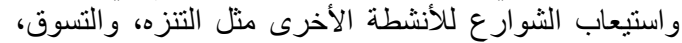
مشاهدة المعالم السياحية، و المناسبات الخاصة.

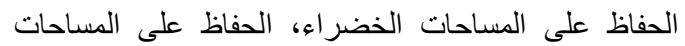
المفتوحة، وخاصة المناطق ذات القيمة البيئية والترفئية التئية

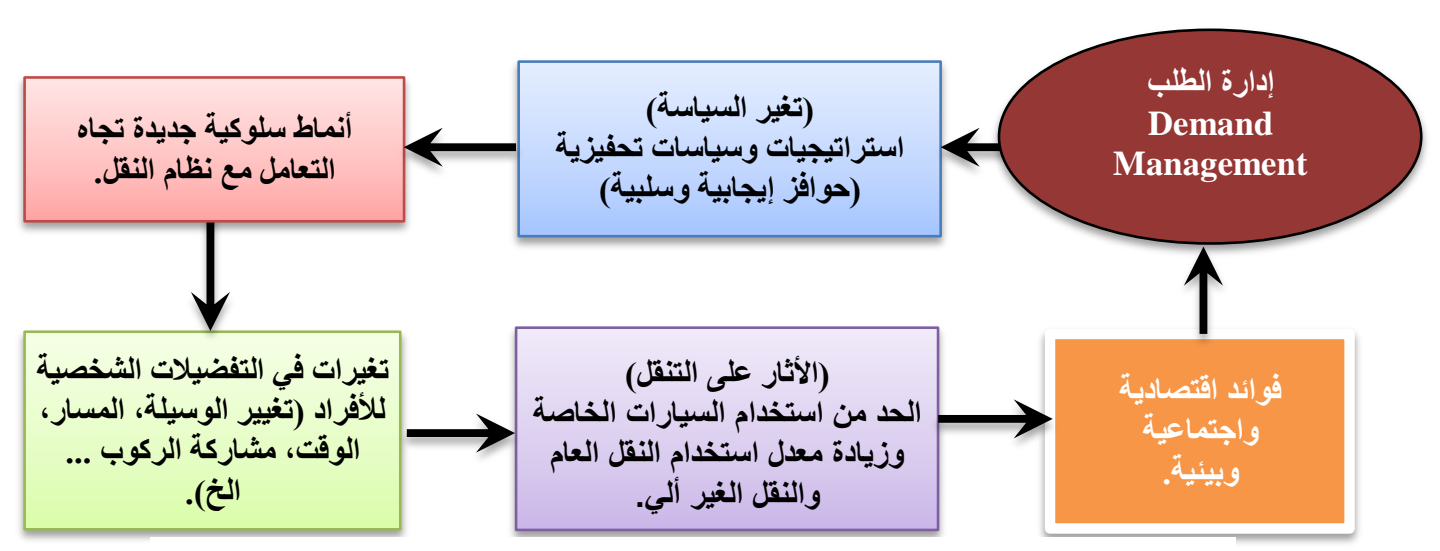

شكل (r) سلسلة أثار إدارة الطلب على النقل علي سلوك التنقل (المصدر: الباحث).

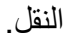

حدود الانبعاثات داخل قدرة البيئة على استيعابها. أ- أثار إدارة الطلب علي سلوك التنقل.

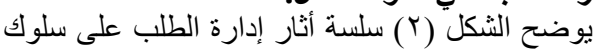

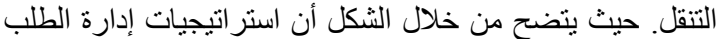

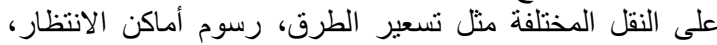

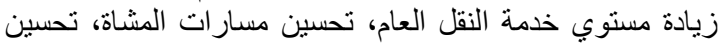

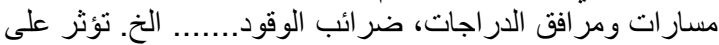

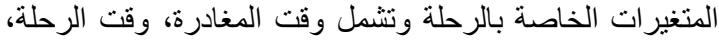

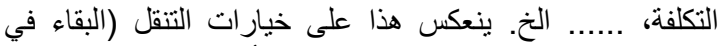

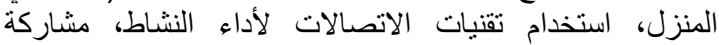

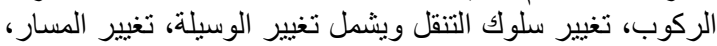

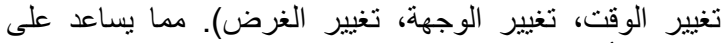

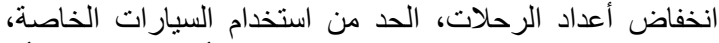

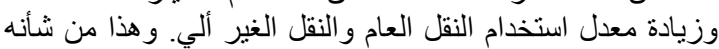

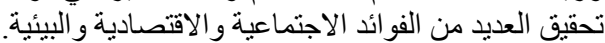

ب- برامج إدارة الطلب المرتبطة بتحقيق الاستدامة في مجال النقل

تتضمن بر امج إدارة الطلب المرتبطة بتحقيق الاستدامة

$$
\text { في مجال النقل الجمع بين ثناثة عناصر (·' ('). }
$$

أو لا: توفير وسائط فعالة ذات جودة عالية تشمل تحسينات خدمة

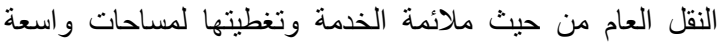

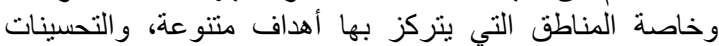
الخاصة بالمشي وركوب الدناط الدرات.
مما سبق يتضح أن النمو الذكي يميل إلي زيادة المشي، التفي،

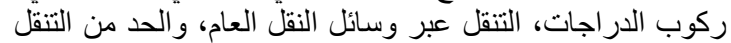

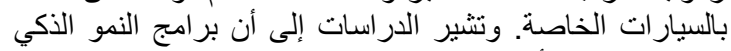

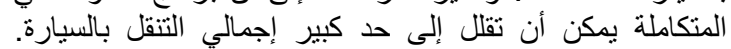

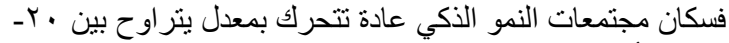

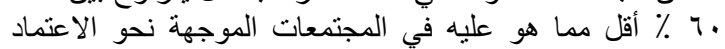
على السيارة. rـ آף عوامل نجاح تدابير إدارة الطلب على النقل لضمان نجاح تدابير إدارة الطلب على النقل، يتطلب

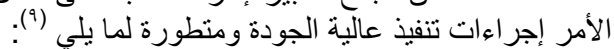
ا. إدارة أماكن انتظار السيارات وبعض الأشكال التكميلية

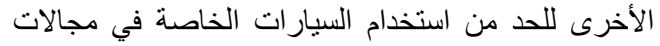

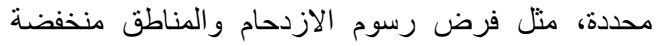

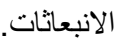
r. توفير وسائل النقل العام عالية الجودة (مثل إصلاحات

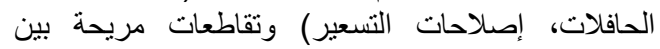

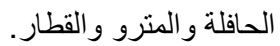

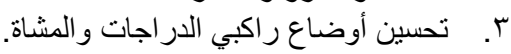

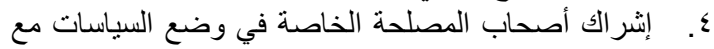

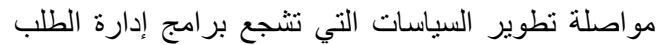

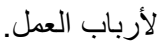


أ .د/ فيصل عبد الدقصود عبد السلام أ.م. د/ إبراهيم حسن هاشم م/ إبر/هيم فتحي الهلطى " نحو أنعاط سلوكية..."

ثالثا: برامج إدارة التنقل وإدارة حركة البضائع: تشمل استخدام

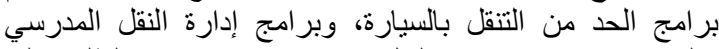

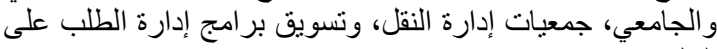

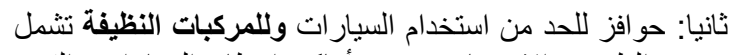

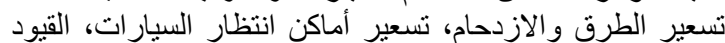

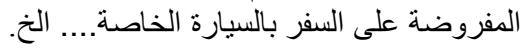

النقل.

\begin{tabular}{|c|c|c|c|c|}
\hline الأدوات التكنولوجية & أدوات المعلومات & الأدوات الاقتصادية & أدوات التظظيم & أدوات التخطيط \\
\hline نظيفة، إنتاج نظيف الوقود، تكنولوجيا أنظمة & 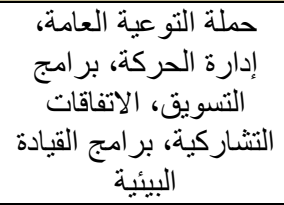 & 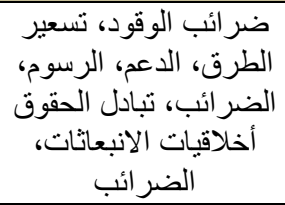 & 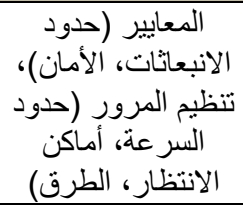 & تخطيط استعمالات الأر اضي المخط العام) \\
\hline
\end{tabular}

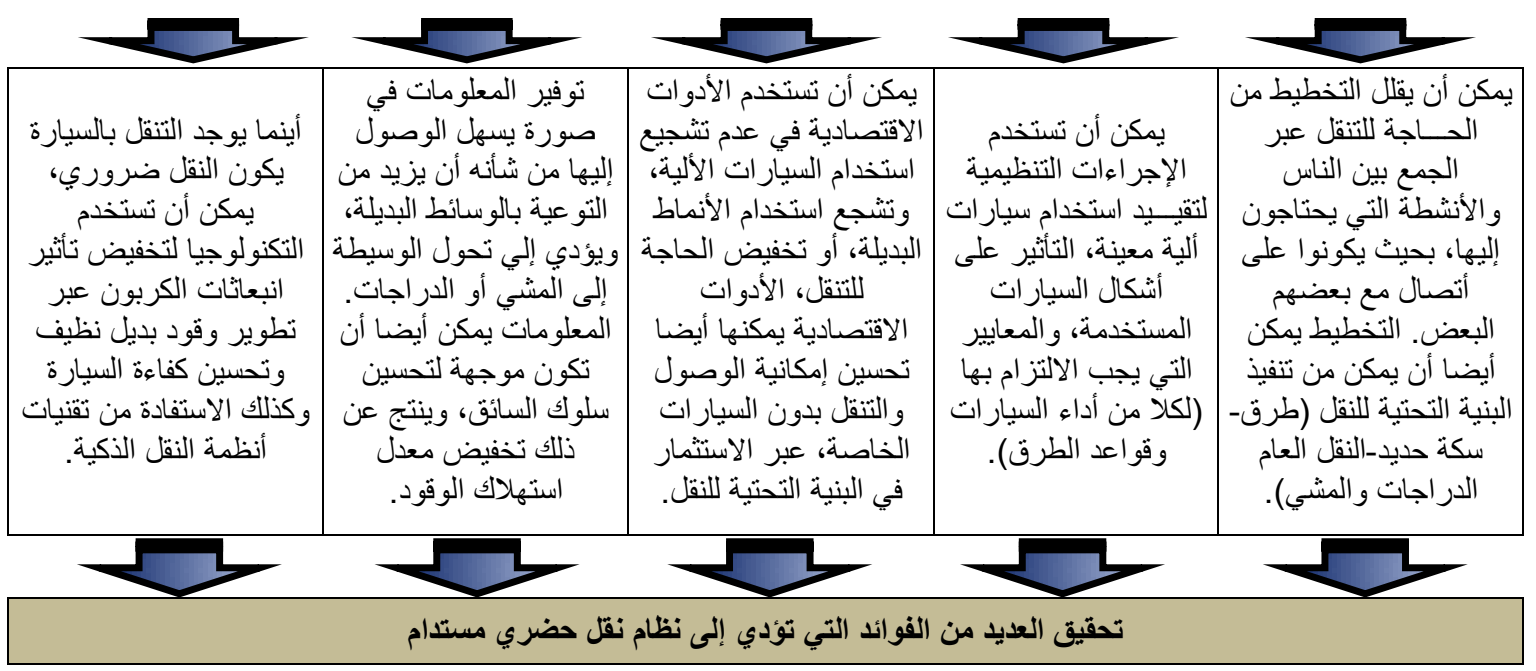

جدول (ء) إدارة الطلب على النقل كجزء من نظام النقل المستدام.

r-1 الأثار المتوقعة لاستراتيجيات إدارة الطلب على عملية التوصيات

• • ربط التخطيط العمراني بقضايا الحركة والنقل والمرور

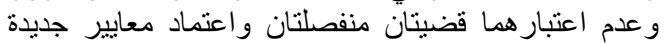

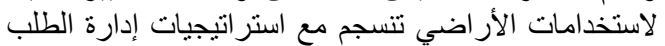
على النقل.

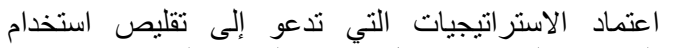
المركبات الخاصة وتفعيل ممر ات الثئ الشئاة و الدر اجات.

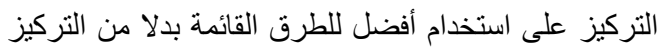

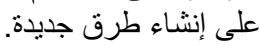

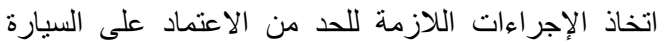

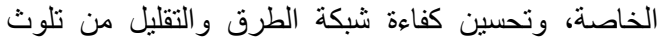

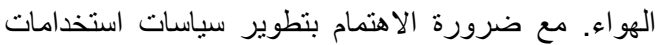

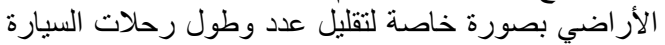
الخاصة والاتجاه نحو الأنماط البديلة (النقل العام). • ت تبني بعض المحفزات الإقناع الأفراد لاستخدام نظام النقل
تتمثل أهم الأثار المتوقعة لاستر اتيجيات إدارة الطلب

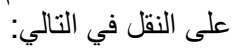

زيادة معدلات استخدام وسائل النقل العام في التنقل.

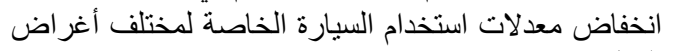

التنقل.

انخفاض في نسبة انبعانات الغاز ات.

زيادة حصنة المشي وركوب انبعان الغاز الدراجات للرحلات ذات المسافات القصيرة (حتى نكم) وكم).

تقلل كفاءة عملية تسعير النظار الأنسار السيارات من مشاكل الانتظار ، وتشجع استخدام الأنماط البديلة.

يقلل من التنقل بالسيارة في أوقات معينة أو في مناطق معينة.

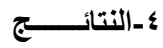

تتمثل المساهمات الرئيسة لإدارة الطلب على النقل لاستدامة نظام النقل في التالي: التمات

تجعل قرارات التنقل الخاصة أكثر فاعلية، عن طريق باتيق تحسين و عي الناس بالخيار ات البديلة. تعظم العائد من الأنفاق على البنئ البنية التحتية. تحافظ على معظم أصول الصغ النقل الحالية.

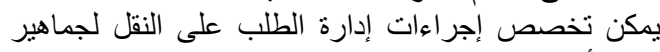
معينة أو جهات بعينها. تتمنل أهم نتائج إدارة الطلب علب على النقل في "أنماط سلوكيه 
أ ـد/ فبصل عبد المقصود عبد السلام أ.م. د/ إبر/هبم حسن هاشم م/ إبر/هيم فتحي الملطى " نحو أنماط سلوكية..."

1 - Jean-Paul Rodrigue, The Geography of Transport Systems, Third edition. New York, 2013, p.355.

2- //www.tc.gc.ca/eng/programs/environmentutsp-tdmintro-1039.htm

$3-$

http://tmip.fhwa.dot.gov/clearinghouse/docs/quick/ Quick.pdf

4 -

http://www.atlantaregional.com/transportation/mo bility-management.(may,2013.).

5 -

http://tmip.fhwa.dot.gov/clearinghouse/docs/quick/ Quick.pdf

6 - Monowar Mahmood, Traffic Management System and Travel Demand Management (TDM) Strategies:, Asian Journal of Management and Humanity Sciences, Vol. 4, No. 2-3, pp. 161-178, 2009.

7- Monowar Mahmood, Traffic Management System and Travel Demand Management (TDM) Strategies:, Asian Journal of Management and Humanity Sciences, Vol. 4, No. 2-3, pp. 161-178, 2009.

8 - GIZ China, Transport Demand Management in Beijing," Reducing Carbon Emissions through Transport Demand Management Strategies", A review of international examples Final report,2012.Pp,19-25

9 - GIZ China, Transport Demand Management in Beijing," Reducing Carbon Emissions through Transport Demand Management Strategies", A review of international examples Final report,2012.P107.

10 - GIZ China, Transport Demand Management in Beijing," Reducing Carbon Emissions through Transport Demand Management Strategies", A review of international examples Final report,2012.Pp,19-25 